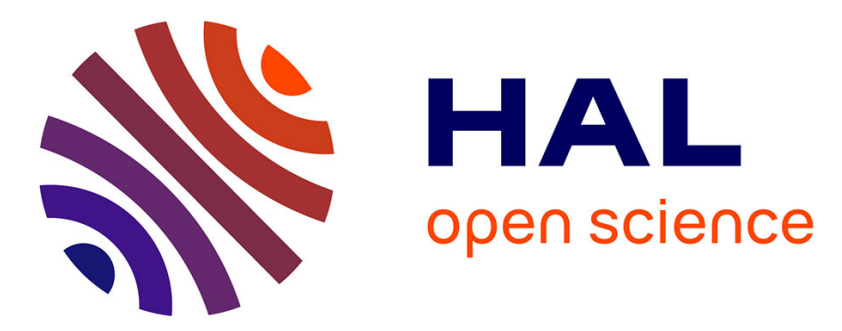

\title{
Les essais inter-laboratoires en hydrométrie, méthodologie et cas d'applications
}

Guillaume Dramais, B. Blanquart, J. Le Coz, G. Pierrefeu, A. Hauet, D. Atmane, K. Pobanz

\section{- To cite this version:}

Guillaume Dramais, B. Blanquart, J. Le Coz, G. Pierrefeu, A. Hauet, et al.. Les essais interlaboratoires en hydrométrie, méthodologie et cas d'applications. La Houille Blanche - Revue internationale de l'eau, 2014, 5/2014 (5), pp.17-23. 10.1051/lhb/2014045 . hal-01191647

\section{HAL Id: hal-01191647 \\ https://hal.science/hal-01191647}

Submitted on 2 Sep 2015

HAL is a multi-disciplinary open access archive for the deposit and dissemination of scientific research documents, whether they are published or not. The documents may come from teaching and research institutions in France or abroad, or from public or private research centers.
L'archive ouverte pluridisciplinaire HAL, est destinée au dépôt et à la diffusion de documents scientifiques de niveau recherche, publiés ou non, émanant des établissements d'enseignement et de recherche français ou étrangers, des laboratoires publics ou privés. 


\title{
Les essais inter laboratoires en hydrométrie, méthodologie et cas d'applications
}

\author{
Guillaume DRAMAIS ${ }^{1}$, Bertrand BLANQUART ${ }^{2}$, Jérôme LE COZ1, Gilles PIERREFEU ${ }^{3}$, \\ Alexandre HAUET ${ }^{4}$, Dihia ATMANE ${ }^{1}$, Karine POBANZ ${ }^{5}$
}

\author{
1. Irstea - UR HH - 5 rue de la Doua, 69626 Villeurbanne - guillaume.dramais@irstea.fr \\ 2. B. Blanquart, Conseil et formation en métrologie, 40 av.du Gal Leclerc54600 Villers les Nancy-bertrand.blanquart@gmail.com \\ 3. CNR, Laboratoire, Direction du Patrimoine Fluvial et Industriel, 1, rue de Chalon-sur-Saône 69007,Lyon-g.pierrefeu@cnr.tm.fr \\ 4.EDF-DTG-DMM - 21 avenue de l'Europe, 38000 Grenoble, France - alexandre.hauet@edf.fr \\ 5. ALTRAN pour CNR Laboratoire, karine-externe.pobanz@cnr.tm.fr
}

\begin{abstract}
RÉSUMÉ. - En parallèle de la méthode de propagation des incertitudes, méthode de référence (GUM - Guide pour l'expression de l'incertitude de mesure) qui nécessite de modéliser l'ensemble du processus de mesure, la méthode expérimentale des essais interlaboratoires présente un grand intérêt pour quantifier les incertitudes d'une méthode de mesure, dans des conditions données. Cette méthode, très utilisée dans certains domaines (chimie, biologie, essais mécaniques, etc.), est encadrée par des normes ISO compatibles avec le GUM décrivant la méthode de référence.

Ainsi, les comparaisons interlaboratoires hydrométriques permettent de quantifier l'incertitude résultant des erreurs de mesure qui s'expriment lors de la répétition de jaugeages simultanés par plusieurs équipes en conditions de répétabilité et de reproductibilité, et en particulier pour une plage de débit constant. Des formules simples permettent de quantifier, à partir des résultats expérimentaux de variance de répétabilité et de variance interlaboratoire, l'incertitude de la méthode de jaugeage testée, supposée non biaisée.

A partir d'exemples récents d'intercomparaisons de jaugeages ADCP, sur perche, par camion et par dilution, des recommandations générales sont proposées pour l'organisation d'intercomparaisons hydrométriques qui soient exploitables pour quantifier des incertitudes conformes aux textes de référence. La méthode normalisée des essais interlaboratoires améliore l'évaluation des incertitudes sur les techniques de jaugeage.
\end{abstract}

Mots-clés : mesure hydrologique, incertitude, mesure en laboratoire, intercomparaison.

\section{Hydrometric inter-laboratory tests, procedure and applications}

\begin{abstract}
In addition to the uncertainty propagation method provided by the GUM (Guide to the expression of uncertainty in measurement) witch requires the modelling of the measurement process, the inter-laboratory experimental method is a good solution to deal with the uncertainty of a measurement technique, in given conditions. This method is commonly frequently used in some fields (eg. in chemistry); it is managed with ISO standards and technical notes or specifications.

Thus, inter-laboratory comparisons in hydrometry allow the quantification of uncertainty due to errors that will be expressed in repeated simultaneous gauging operations. These repetitions have to be done by different teams, at the same time, in stable flow conditions, under repeatability and reproducibility conditions. Simple formulae with repeatability variance and the inter-laboratory variance results assessed to evaluate the gauging technique uncertainty, supposed to be unbiased. The bias or systematic error is linked to the gauging technique; it could be evaluated by comparison with an independent flow measurement technique with a known uncertainty.

In this study some suggestions are presented for the hydrometric inter-laboratory comparison organisation. These pieces of advice follow from recent inter-laboratory comparison with ADCP, current meter on wading rod or gauging van, and tracer dilution devices. The inter-laboratory comparison method is a standardised solution for improving the knowledge and the uncertainty evaluation of gauging techniques.
\end{abstract}

Key-words: hydrometric, uncertainty, laboratory measurement, inter-laboratory comparison.

\section{INTRODUCTION}

La mesure du débit des rivières est complexe, principalement du fait de la morphologie variée des sections de jaugeage, de la distribution des vitesses dans ces sections et de la variabilité temporelle du débit. L'étalonnage de certains capteurs utilisés en hydrométrie est possible. Ainsi, les moulinets hydrométriques peuvent être comparés à la vitesse de déplacement d'un chariot porteur sur un canal d'eau stagnante [ISO, 2007] (Figure 1 et 2), une perche de courantomètre peut être vérifiée par un mètre raccordé aux étalons internationaux, une cellule de conductimètre peut être testée et étalonnée avec des solutions salines connues, le volume d'un seau peut être déterminé par pesée avec une balance de précision adaptée. Ces vérifications et contrôles sont indispensables pour la justesse des mesures de vitesse, de section, ou autres. Seuls des étalonnages dans la grandeur « débit » permettraient d'accéder à l'incertitude d'un jaugeage par 


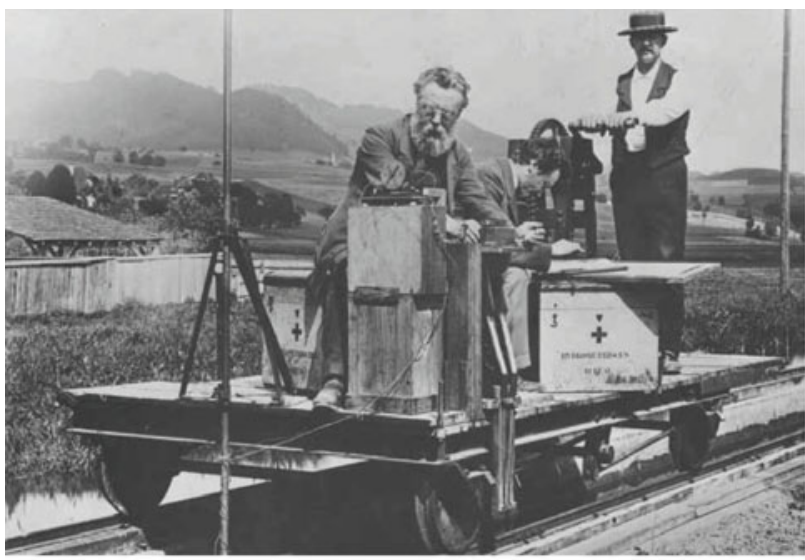

Figure 1 : Josef Epper et le premier chariot d'étalonnage de moulinets hydrométriques (1896) [Metinfo, 2012].

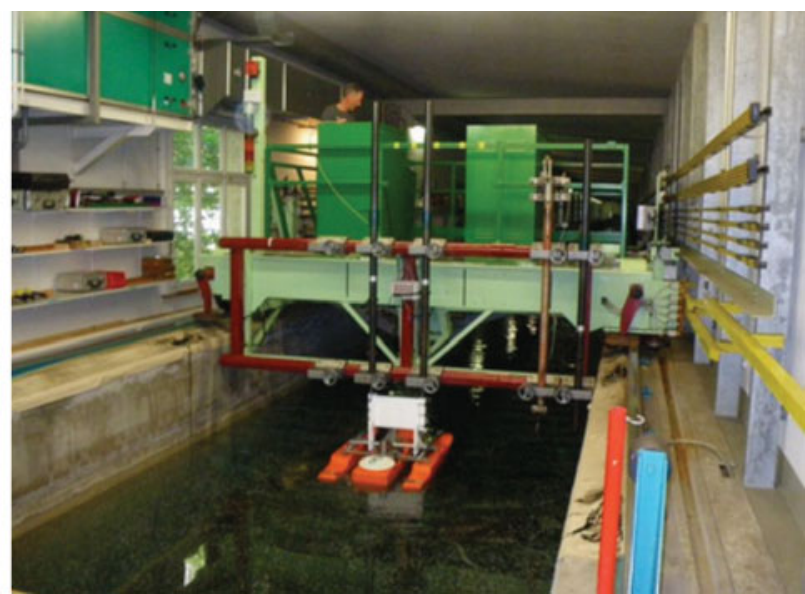

Figure 2 : Canal actuel d'étalonnage pour courantomètres du Metas (Office fédéral de métrologie en Suisse). une méthode identifiée sans hypothèse contraignante sur la répartition des vitesses d'écoulement ou la durée de mesure.

L'évaluation d'une incertitude de mesure conforme au GUM sur un jaugeage est possible pour certaines techniques. Par exemple la technique par dilution de traceur est bien documentée [ISO, 1994a], la norme sur la mesure par exploration du champ de vitesse à l'aide d'un moulinet [ISO, 2009] propose aussi un calcul d'incertitude par propagation, d'autres travaux viennent enrichir cette approche [Le Coz, 2012a]. L'évaluation des incertitudes sur le jaugeage par profileur de courant à effet Doppler, qui s'est imposé comme la technique de référence sur les grands cours d'eau depuis quelques années, est un sujet d'étude complexe. Les mesures effectuées par ces différents capteurs ne sont pas raccordées aux étalons et les erreurs liées à l'environnement de mesure et à la qualité du déploiement sont difficilement quantifiables. Par conséquent, l'application de la méthode par propagation des incertitudes est impossible sans faire de nombreuses hypothèses sur certaines composantes d'incertitude.

Cependant les exigences de qualité et de fiabilité des mesures devenant incontournables, les résultats de mesure doivent être exprimés avec une incertitude. L'approche expérimentale par comparaison interlaboratoire est alors une bonne alternative pour évaluer l'incertitude de mesure sur les méthodes de jaugeage.

Par ailleurs, il convient de remarquer que les laboratoires d'étalonnage ou d'essais, dans lesquels l'estimation des incertitudes peut être réalisée par application directe du GUM, sont soumis à l'organisation de comparaisons interlaboratoires pour valider leurs estimations d'incertitude, que ce soit au niveau des laboratoires nationaux de métrologie comme au niveau des laboratoires accrédités.

\section{METHODOLOGIE DES ESSAIS INTERLABORATOIRES EN HYDROMETRIE}

\section{II.1. Généralités}

Les équipes d'hydrométrie ont depuis longtemps cherché à valider leurs méthodes et leurs instruments de mesure en comparant les capteurs, ou les méthodes entre elles. Ces essais ont la plupart du temps pour but d'appréhender l'aptitude des équipes a travers leur mode opératoire et leur instrumentation à obtenir des résultats de mesure de débit conformes à une valeur de référence. En hydrométrie, la difficulté est qu'il n'existe pas de valeur de référence pour le débit raccordée aux étalons internationaux. Les équipes comparent donc leurs résultats entre elles, à une donnée issue d'une relation hauteur-débit, ou encore aux résultats donnés par une mesure consensuelle estimée plus fiable. Ce type d'approche est rassurant pour les jaugeurs, donne confiance dans le matériel, les méthodes mais ne permet pas d'évaluer l'incertitude. Des comparaisons interlaboratoires d'ADCP (Acoustic Doppler Current Profiler) ont lieu régulièrement dans le monde de l'hydrométrie, par exemple en Angleterre [Everard, 2009], en Croatie [Terek et al., 2008] et en France depuis 2009 sous l'impulsion du Groupe Doppler [Le Coz et al., 2007] et sont transposables aux autres techniques de jaugeage comme les courantomètres et les moulinets sur perche [Dramais et al., 2011]. Les traitements statistiques de ces essais permettent d'utiliser ces comparaisons interlaboratoires comme outil d'évaluation des incertitudes [ISO 21748].

\section{II.2. Cadre général}

Les essais interlaboratoires peuvent avoir trois objectifs différents :

- Attribuer une valeur consensuelle à une caractéristique (par exemple, calculer avec une incertitude réduite le débit d'une rivière en l'absence de mesure de référence raccordée aux étalons internationaux) ;

- Déterminer la performance d'une méthode (par exemple comme dans les comparaisons du Groupe Doppler, évaluer les performances de la méthode de jaugeage par ADCP mobile). C'est l'objectif suivi dans la plupart des comparaisons interlaboratoires en hydrométrie et qui fait l'objet de la méthode présenté dans cet article ;

- Déterminer l'aptitude d'un laboratoire ou d'un instrument, c'est-à-dire leur conformité à des spécifications données.

Selon l'objectif poursuivi, les essais réalisés et leurs modalités pratiques d'organisation pourront être très différents (choix du site, réalisation simultanée ou différée des mesurages, etc.) ; il est donc nécessaire de bien définir l'objectif prioritaire du test interlaboratoire. Ici, le second objectif est recherché. 


\section{II.3. Déroulement du calcul}

L'exploitation et l'analyse des résultats des essais interlaboratoires vont permettre d'évaluer expérimentalement l'incertitude de la méthode de mesure dans les conditions de mesure de la comparaison.

Pour les essais interlaboratoires en hydrométrie présentés dans ce travail, trois référentiels normatifs ont été utilisés :

- La norme ISO 5725-2 [ISO, 1994b] a pour objectif de quantifier les performances de la méthode, en termes de répétabilité et de reproductibilité ;

- La norme ISO 21748 [ISO, 2010] permet de traduire les résultats précédents en termes d'incertitude de mesure ;

- La norme ISO 13528 [ISO, 2005] permet de déterminer l'incertitude associée au débit de référence calculé sur l'ensemble des laboratoires. Cette démarche ne permet pas de déterminer une caractéristique de performance des laboratoires d'un point de vue général.

Les hypothèses nécessaires à l'application des deux premières normes sont les suivantes :

- Les résultats expérimentaux sont obtenus dans des conditions dans lesquelles les principales composantes d'erreur ont la possibilité de s'exprimer ;

- Les résultats sont indépendants les uns des autres et les erreurs de mesure suivent une loi de distribution gaussienne (ou à défaut au moins unimodale) ;

- La norme ISO 5725-2 fait l'hypothèse que la répétabilité est identique pour toutes les équipes et, dans une moindre mesure, que le nombre d'essais par débit est identique pour toutes les équipes.

Les principales étapes de calcul sont les suivantes (tous les calculs sont détaillés dans les documents de l'ISO et il est possible de les implémenter dans un tableur [Atmane, 2012]) :

- Recueil et mise en forme des données;

- Examen des valeurs ;

- Calcul des statistiques de Mandel pour examen visuel de l'homogénéité des résultats ;

- Détection des valeurs aberrantes (mise en œuvre des tests de Grubbs et Cochran), ces tests sont facultatifs mais peuvent êtres réalisés en cas de doute suite à l'examen des résultats des statistiques de Mandel ;

- Ensuite il convient de calculer les différents écart-types de répétabilité $\left(S_{r}\right)$, interlaboratoire $\left(S_{L}\right)$ et de reproductibilité $\left(S_{R}\right)$.

La variance de répétabilité $S_{r}$ est obtenue par la formule suivante :

$$
s_{r}^{2}=\frac{\sum_{i=1}^{P}\left(n_{i}-1\right) s_{i}^{2}}{\sum_{i=1}^{P}\left(n_{i}-1\right)}
$$

Avec $i$ l'indice de l'équipe, $S_{i}$ l'écart type expérimental et $n_{i}$ le nombre de répétition des mesures de débit $Q_{i, k}$ fournies par chaque équipe, $k$ étant l'indice de la mesure.Dans le cas où $n_{i}=2$, les documents normatifs indiquent que l'écart type expérimental est donné par :

$$
s_{i}=\frac{\left|Q_{i, 2}-Q_{i, 1}\right|}{\sqrt{2}}
$$

La variance interlaboratoire $S_{L}$ est obtenue par la formule :

$$
s_{L}^{2}=\frac{s_{d}^{2}-s_{r}^{2}}{N}
$$

Avec $N$ le nombre moyen de mesures fournies par équipe. L'écart type de la moyenne des équipes $S_{d}$ est donné par :

$$
S_{d}^{2}=\frac{1}{p-1} \sum_{i=1}^{n} n_{i}\left(\bar{Q}-Q_{\text {mean }}\right)^{2}
$$

Pour $P$ instruments de mesure.

La variance de reproductibilité est obtenue par :

$$
s_{R}^{2}=s_{L}^{2}+s_{r}^{2}
$$

avec $s_{L}^{2}$ la variance interlaboratoire qui caractérise la dispersion des débits moyens entre les équipes,

et $s_{r}^{2}$ la variance de répétabilité qui caractérise la dispersion des débits répétés pour une équipe autour de sa propre moyenne.

Si les hypothèses sont respectées, et en particulier si toutes les sources d'erreur ont pu s'exprimer au cours de la comparaison, alors on peut écrire que l'estimation de l'incertitude associée à une observation issue de l'essai interlaboratoire traité est :

$$
u\left(Q_{1,1}\right)=s_{r}
$$

avec $Q_{1,1}$ le résultat expérimental fourni par un laboratoire et pour une observation.

Ce qui conduit à l'expression de l'incertitude élargie $(\mathrm{k}=2)$ :

$$
U\left(Q_{1,1}\right)=2 . s_{r}
$$

Dans le cas des jaugeages par ADCP, si on veut calculer l'incertitude de la moyenne de $n$ jaugeages ADCP, réalisés par $p$ instruments, en prenant en compte si possible le biais de la méthode (cf. II.4) il est possible d'utiliser la formule suivante [Le Coz, 2012b] :

$$
U\left(Q_{n, p}\right)=2 \sqrt{u_{\text {biais }}^{2}+\frac{s_{r}^{2}}{n p}+\frac{s_{L}^{2}}{p}}
$$

Avec $u_{\text {biais }}$ l'incertitude liée au biais de la méthode ADCP.

\section{II.4. Expression du biais}

Il est à noter qu'on évalue ainsi l'incertitude, non pas sur une mesure donnée, mais sur une méthode ${ }^{1}$ de mesure, appliquée par l'ensemble des équipes de façon homogène et dans un environnement similaire, avec un certain nombre de sources d'erreur laissées libres, et qui vont donc s'exprimer dans la variabilité des résultats. En revanche, les erreurs systématiques liées à la méthode de mesure seront communes à toutes les équipes. Le biais, ou erreur systématique est lié à la méthode de jaugeage choisie. Par exemple dans le cas des comparaisons de mesures ADCP, le choix commun à toutes les équipes de formules d'extrapolation identiques, ne s'exprime donc pas dans l'intercomparaison, et

1. La «méthode » est ici le protocole de déploiement d'un certain type d'instruments dans une configuration de rivière donnée, par exemple " méthode de jaugeage des cours d'eau à pied par courantomètres sur perche » ou " méthode de jaugeage des cours d'eau par profileur de courant mobile en mode transect ». 
donc ne se retrouve pas dans les résultats expérimentaux. Le biais de la méthode peut être approché par des tests de sensibilité aux paramètres ou par comparaison à une mesure de débit indépendante, d'incertitude maîtrisée et connue, prise comme référence. Par exemple dans les travaux de la CNR [Olivier et al., 2008] sur une comparaison de 19 jaugeages au moulinet pris comme référence avec des données de jaugeages par ADCP $1200 \mathrm{KHz}$ sur une gamme étendue de débit (de $11 \mathrm{~m}^{3} / \mathrm{s}$ à $3500 \mathrm{~m}^{3} / \mathrm{s}$ correspondant à des vitesses d'écoulement entre $5 \mathrm{~cm} / \mathrm{s}$ et $2,5 \mathrm{~m} / \mathrm{s}$ ) avait permis d'évaluer l'incertitude des jaugeages ADCP entre 3 et $6 \%$ dans les conditions de la comparaison. Dans cette étude comparative, le biais de la méthode par ADCP mobile avait été évalué : $u_{\text {biais }}=0,5$.

\section{EXEMPLES D’APPLICATION}

Ces dernières années, plusieurs comparaisons interlaboratoires ont été organisées et exploitées avec succès par différents groupes de travail en France. Ainsi le Groupe Doppler formé en 2005 a été l'initiateur dans ce domaine en organisant les premières comparaisons inter-organismes de mesure de débit par profileurs de courant à effet Doppler (ADCP) en France :

La Vézère à Allassac en 2009 [Le Coz et al., 2009]. Cette campagne a réuni 21 équipes pour déployer des profileurs de courant sur supports autonomes ou tractés depuis les berges. Quatre essais ont eu lieu avec des consignes de déploiement différentes (paramétrage libre ou imposé, cadence de mesure libre ou imposée). Le débit était stabilisé par demi-journée par le barrage situé en amont du site.

Le Rhône à Génissiat en octobre 2010 [Pobanz et al., 2011] et septembre 2012 (essai en cours d'analyse). Ces comparaisons concernent 15 à 30 équipes déployant des profileurs de courant depuis des bateaux. Le site de Génissiat offre plusieurs tronçons différents au niveau morphologique et un ensemble barrage/usine permettant la réalisation de plusieurs paliers de débits constants. Les conduites de l'usine sont munies d'un système de mesure du débit par ultrasons à temps de transit, avec une incertitude réduite ( $1 \%$ selon le constructeur, $3 \%$ selon une évaluation d'incertitude conforme au GUM). Ce site est donc idéal pour ce genre d'essai sur grands cours d'eau.
Le canal de la Gentille en septembre 2011 [Hauet et al., 2012] (fig. 4). Cette intercomparaison a réuni une vingtaine d'équipes pour déployer des profileurs de courant sur supports tractés depuis les berges du canal. Ce site est un canal trapézoïdal qui a permis des comparaisons de méthodes intéressantes et des calculs complémentaires sur les profils de vitesse et les lois d'extrapolation. L'instabilité du débit (manœuvres d'usine dues à une situation d'étiage sévère) a malheureusement nui aux essais.

Le Réseau Mesure d'Irstea créé en 2002 rassemble des métrologues, techniciens et chercheurs de l'institut dans des ateliers thématiques. L'atelier débitmétrie liquide à surface libre est le plus ancien du Réseau Mesure et ses travaux en métrologie l'ont amené naturellement à organiser aussi une comparaison interlaboratoire. Cette intercomparaison (fig. 3) a porté sur les jaugeages au courantomètre sur perche de 11 équipes, sur l'Ouvèze et le Toulourenc, en mai 2011 [Dramais et al., 2011]. Les courantomètres utilisés étaient mécaniques (moulinets), électro-magnétiques ou acoustiques Doppler. Chaque équipe a réalisé deux jaugeages successifs sur chaque cours d'eau avec un protocole imposé. Des dépouillements avec différents nombres de verticales et de points sur la verticale ont pu être évalués notamment la méthode dite " japonaise " qui privilégie la description de la géométrie de la section et qui limite le nombre de points de vitesses afin de gagner du temps de jaugeage [Le Coz et al., 2012a]. Cet essai a confirmé la difficulté de jauger les petits débits $\left(\sim 1 \mathrm{~m}^{3} / \mathrm{s}\right)$, en montrant des incertitudes plus fortes que celles attendues dans les bonnes conditions d'application de la technique. Là encore, l'instabilité du débit a nui aux résultats d'évaluation des incertitudes.

Différentes équipes comme les services de l'Etat ou la CNR organisent localement des comparaisons interlaboratoires très riches d'enseignements et de résultats intéressants (ADCP embarqués à Chateauneuf-du-Rhône en 2009, ADCP tractés à Fontaine de Vaucluse [Atmane, 2012], ADCP tractés à Chooz en 2012, camions jaugeurs à Vic le Comte en 2011). Enfin ces essais interlaboratoires associés à des plans d'expérience bien définis peuvent permettre aux équipes de tester d'autres mesurandes et leurs paramètres d'influence dans des conditions environnementales plus stables que sur le terrain, comme la vitesse de l'eau en canal [Chaumont, 2011], ou la fluorescence en laboratoire [Atmane, 2012].

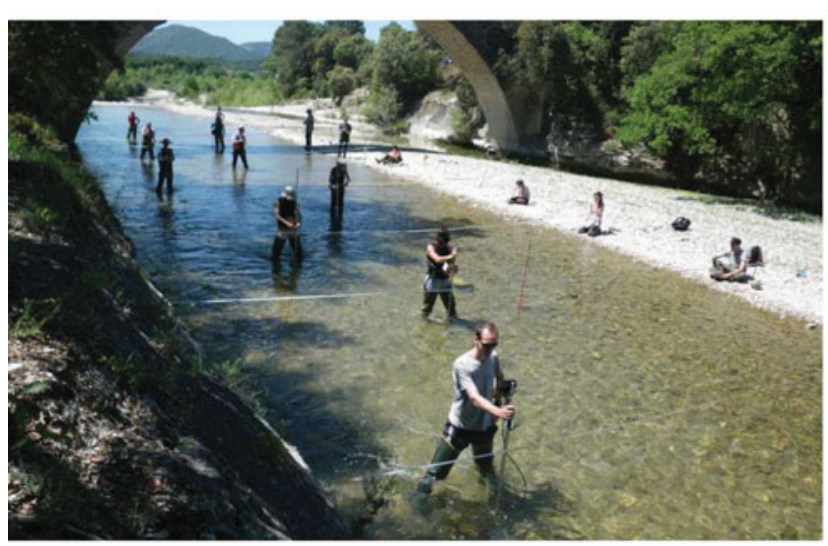

Figure 3 : Les courantomètres sur perche déployés sur l'Ouvèze à Entrechaux (2011).

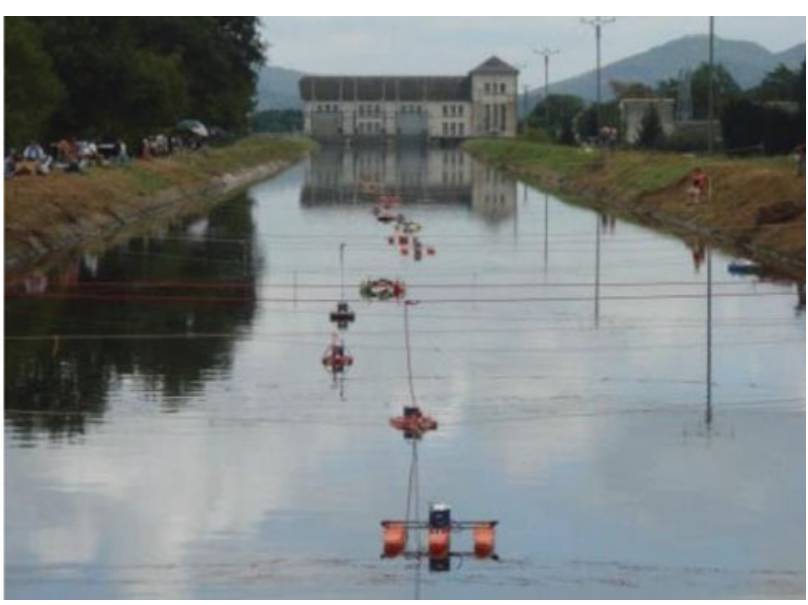

Figure 4 : Des capteurs ADCP déployés sur le canal de la Gentille (2011). 


\section{RESULTATS ET ENSEIGNEMENTS}

\section{IV.1. Exploitation des mesures}

Le tableau 1 présente un échantillon de données issues des campagnes d'essai interlaboratoires de mesures de débit qui ont été traitées par la méthode présentée dans cette étude. Ici, par "laboratoire " on entend chaque capteur indépendant déployé suivant la méthode de jaugeage déterminée lors de la préparation de la campagne. Chaque mesure est définie comme la moyenne de 4 ou 6 traversées ADCP selon les essais. Les résultats des évaluations d'incertitude sur les techniques de mesure varient entre 4 et $12 \%$ selon les essais, et le site pour les campagnes mettant en jeu les ADCP et jusqu'à $16 \%$ pour les essais sur l'Ouvèze et le Toulourenc avec des courantomètres sur perches. Les bornes hautes et basses d'incertitudes correspondent à des traitements sur différentes séries de mesures. Ces séries, réalisées lors d'une même campagne de mesure, diffèrent par la stratégie de déploiement ou de paramétrage (Vézère), la morphologie du transect (Génissiat), les séries de mesures prisent en compte (Gentille), le nombre de laboratoires considérés (Vaucluse). Dans ces différents résultats, le biais de la méthode n'est pas pris en compte.

Pour une technique donnée (en particulier pour le cas des jaugeages par ADCP mobiles), il est confirmé que les conditions de mesure sur la section choisie influencent en premier lieu la qualité de la mesure, au-delà de l'instrumentation et du protocole de mesure. Cette piste est aussi avancée pour expliquer les fortes incertitudes calculées pour l'Ouvèze et le Toulourenc. Dans le cas des ADCP, la réduction de l'incertitude obtenue en moyennant plusieurs transects successifs, voire plusieurs appareils, a pu être quantifiée (figures 5 et 6). Ce type de résultats est cohérent avec l'expérience des jaugeurs et encourage les efforts dans ce sens. En retour, de telles évaluations expérimentales des incertitudes, valables dans les conditions de l'essai, permettent de conforter les incertitudes obtenues par propagation, toujours délicates à implémenter en hydrométrie.

\section{IV.2. L'exemple de Génissiat}

On peut appliquer la formule 4 aux données de la comparaison de Génissiat en 2010 (figures 5 et 6) pour laquelle une valeur du biais de la méthode de jaugeage par ADCP mobile a été évalué à $u_{\text {biais }}=1,25$ par analyse de sensibilité et comparaison à la mesure par ultra-sons dans les conduites de l'usine. Les valeurs d'incertitude déterminées pour l'intercomparaison de Génissiat 2010 étaient très variables selon le site de mesure, qu'il présente des conditions très favorables (amont du pont de Pyrimont) ou défavorables (aval du barrage/usine de Génissiat). A Pyrimont, l'incertitude élargie (au niveau de confiance $95 \%$ ) était de $7 \%$ pour 1 transect et de 5,5\% pour 6 transects en prenant en compte le biais, ces résultats sont conformes aux résultats des travaux de la CNR [Olivier et al., 2008]. A Génissiat, l'incertitude élargie (au niveau de confiance $95 \%$ ) était de $12,2 \%$ pour 1 transect et de $9 \%$ pour 6 transects en prenant en compte le biais. Cette forte incertitude s'explique par une section hydraulique non optimale d'un point de vue mesures mais utile pour la démonstration. Rappelons que les valeurs d'incertitude obtenues ne sont valables que dans les conditions de mesure des essais.

La figure 5 nous confirme le fait que plus on réalise de traversées plus l'incertitude de mesure est réduite, avec un gain réduit à partir de 4 ou 6 transects, d'où les recommandations usuelles. Cette incertitude diminue encore plus rapidement si on utilise plusieurs appareils simultanément.

Tableau 1 : Exemples de campagnes d'essais traitées avec la méthode de comparaison interlaboratoire (la mesure par ADCP est ici la moyenne de 6 traversées successives sauf pour Génissiat 2010 avec 4 traversées successives moyennées).

\begin{tabular}{|c|c|c|c|c|c|c|}
\hline Campagne & $\begin{array}{c}\text { Type } \\
\text { d'instrument } \\
\text { testé }\end{array}$ & $\begin{array}{c}\text { Gamme } \\
\text { de débit } \\
{\left[\mathrm{m}^{3} / \mathbf{s}\right]}\end{array}$ & $\begin{array}{l}\text { Nombre } \\
\text { d'équipes }\end{array}$ & Mesure de contrôle & $\begin{array}{c}\text { Incertitude } \\
\text { sur la méthode } \\
95 \%(\mathbf{k}=\mathbf{2})\end{array}$ & Source \\
\hline Vézère, 2009 & $\begin{array}{c}\text { ADCP tractés } \\
\text { depuis les berges }\end{array}$ & 30 & 37 & $\begin{array}{l}\text { Courbe de tarage } \\
\text { Mesure par dilution } \\
\text { de Rhodamine WT }\end{array}$ & $\pm 4-9 \%$ & $\begin{array}{c}\text { [Le Coz et al., } \\
\text { 2009] }\end{array}$ \\
\hline $\begin{array}{l}\text { Génissiat, } 2010 \\
\text { (Pyrimont) }\end{array}$ & ADCP sur bateau & $110-430$ & 26 & $\begin{array}{l}\text { Courbe de tarage } \\
\text { Temps de transit } \\
\text { en conduite }\end{array}$ & $\pm 4-6 \%$ & $\begin{array}{c}\text { [Pobanz et al., } \\
\text { 2011] }\end{array}$ \\
\hline $\begin{array}{c}\text { Génissiat, } 2010 \\
\text { (Aval } \\
\text { barrage/usine) }\end{array}$ & ADCP sur bateau & $110-430$ & 26 & $\begin{array}{l}\text { Courbe de tarage } \\
\text { Temps de transit } \\
\text { en conduite }\end{array}$ & $\pm 8-12 \%$ & $\begin{array}{c}\text { [Pobanz et al., } \\
\text { 2011] }\end{array}$ \\
\hline Gentille, 2011 & $\begin{array}{c}\text { ADCP tracté } \\
\text { depuis les berges }\end{array}$ & $10-20$ & 34 & $\begin{array}{l}\text { Abaques de rendement } \\
\text { de l'usine } \\
\text { Jaugeage par } \\
\text { exploration des vitesses }\end{array}$ & $\pm 6-7,5 \%$ & $\begin{array}{l}\text { [Hauet et al., } \\
\text { 2012] }\end{array}$ \\
\hline $\begin{array}{l}\text { Ouvèze } \\
\text { Toulourenc, } \\
2011\end{array}$ & $\begin{array}{l}\text { Courantomètres } \\
\text { sur perches }\end{array}$ & 1 & 11 & $\begin{array}{l}\text { Courbe de tarage } \\
\text { Mesure par dilution } \\
\text { de Rhodamine WT }\end{array}$ & $\pm 16 \%$ & $\begin{array}{c}\text { [Dramais et al., } \\
\text { 2011] }\end{array}$ \\
\hline Chooz, 2012 & $\mathrm{ADCP}$ & 35 & 4 & & $\pm 6 \%$ & [Atmane, 2012] \\
\hline Vaucluse, 2012 & $\begin{array}{c}\text { ADCP tractés } \\
\text { depuis les berges }\end{array}$ & 6 & 5 & Courbe de tarage & $\pm 8-10 \%$ & [Atmane, 2012] \\
\hline
\end{tabular}




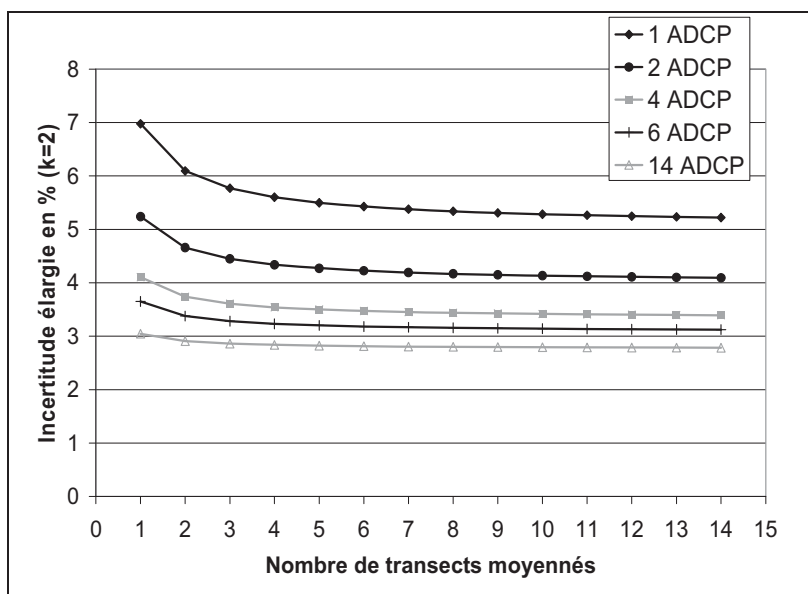

Figure 5 : Intercomparaison de Génissiat 2010 - Site de Pyrimont - Incertitude élargie généralisée pour $N$ transects et $p$ ADCP - Biais compris (cf. formule 8).

Quand la prise en compte du biais de la méthode est possible, les incertitudes élargies sont plus réalistes. En effet lorsque le biais est négligé (figure 6) les niveaux d'incertitudes sont sous-estimés.

Il est à noter que l'écart entre la moyenne de l'ensemble des ADCP face à la mesure ultra-son en conduite est comprise entre 1 et $3 \%$ selon le palier de débit concerné, les mesures ADCP étant toujours au dessus des valeurs ultra-son. Ce constat de 2010 semblerait se retrouver en 2012 selon les dépouillements « réalisés à chaud». Cette valeur est tout à fait compatible avec l'incertitude attendue du système ultra-son sans qu'on puisse différencier quelle serait la valeur la plus juste.

La synthèse des résultats et des conditions de ces tests interlaboratoires réalisés en France depuis 2009 permet d'apporter quelques pistes pour l'organisation et l'exploitation de prochaines campagnes.

\section{IV.3. Organiser une campagne de comparaison interlaboratoire en hydrométrie}

Afin de réaliser l'essai le plus utile possible, il est nécessaire de bien définir l'objectif (cf. § II.2) : essai d'aptitude, évaluation d'une méthode ou détermination d'une valeur consensuelle (ce dernier cas impose la plupart du temps le site exploré). Dans les deux premiers cas, il faut trouver un site adapté aux contraintes de déploiement de la technique ou des capteurs que l'on veut évaluer. Le site choisi doit offrir les mêmes caractéristiques environnementales à tous les participants (morphologie de la section, conditions amont et aval). Le nombre de participants doit être suffisant $(>3)$ et adapté au site. Le mesurande, c'est-à-dire la grandeur physique à mesurer, doit être rigoureusement défini. Il s'agit en général du débit du cours d'eau supposé parfaitement constant durant la plage de mesure et égal sur toutes les sections du site de mesure. Cette condition est la principale à vérifier pour réussir une intercomparaison. Il faut également définir comment est établie cette mesure, en particulier si elle est la moyenne de plusieurs réplicats, comme c'est le cas pour la mesure par ADCP. L'expérience montre que la stabilité du débit est difficile à obtenir, la mise en place d'échelle limnimétrique et d'un enregistreur provisoire est indispensable (un protocole d'essai en mode dégradé doit être prévu). Au lieu du débit, l'intercomparaison peut aussi

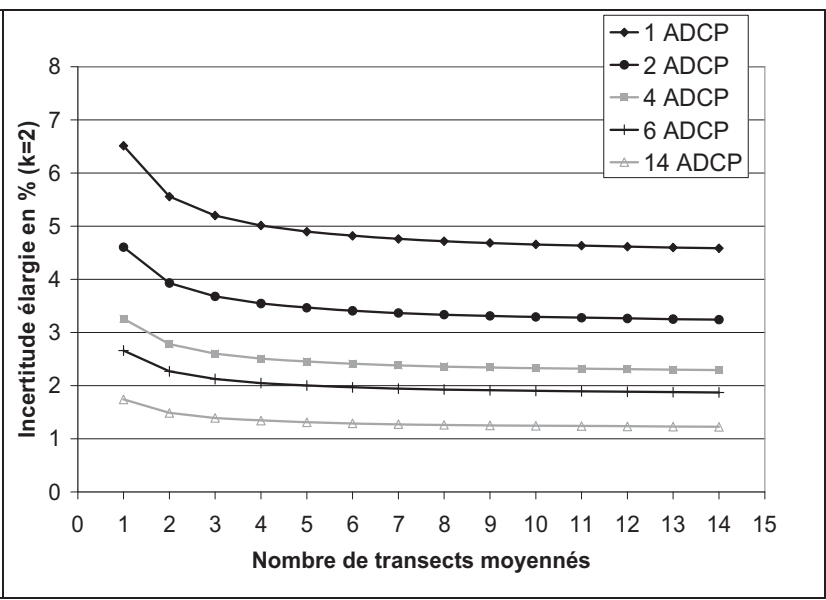

Figure 6 : Intercomparaison de Génissiat 2010 - Site de Pyrimont - Incertitude élargie généralisée pour $N$ transects et p ADCP - Biais négligé (cf. formule 8).

porter sur une mesure élémentaire importante pour l'établissement du débit, comme la vitesse, la profondeur, un angle d'inclinaison, la température, la concentration en traceur, etc. Le protocole de l'essai doit être détaillé (logistique, sécurité des opérateurs, contraintes de déploiement, déroulement, choix des paramètres d'influence testés, formats de fichiers de mesure, synchronisation de tous les instruments de mesure). En s'organisant bien et collectivement, des résultats à chaud peuvent être fournis rapidement aux participants.

\section{CONCLUSIONS}

Les essais interlaboratoires permettent une estimation expérimentale de l'incertitude de mesure pour la méthode de jaugeage testée dans les conditions du jour.

Ces essais sont utiles à plusieurs niveaux. Tout d'abord ils permettent aux participants de partager leurs expériences, d'harmoniser les pratiques entre les services. Ensuite au niveau métrologique, confronter un capteur dans une intercomparaison est un contrôle et une validation de la fiabilité de cet instrument.

En l'absence d'étalon pour la mesure de débit à surface libre, ces essais sont indispensables pour garantir la qualité des données, de plus ils permettent de fournir une valeur consensuelle d'incertitude par comparaison avec des méthodes de mesures reconnues fiables même si elles ne sont pas totalement raccordées d'un point de vue métrologique (débit fourni par un équipement ultrason en conduite ou par méthode thermodynamique).

D'un point de vue plus général, multiplier les comparaisons dans des configurations variées d'écoulement par des méthodes différentes contribue à améliorer notre connaissance de l'incertitude de mesure sur ces méthodes d'hydrométrie, notamment dans la perspective de valider le développement de méthodes de calcul des incertitudes applicables à tout jaugeage. En parallèle réitérer les intercomparaisons ADCP sur des sites équipés des systèmes de mesure en conduite (mesure ultra-son sur le site CNR de Génissiat, ou mesures thermodynamiques sur une usine de haute chute d'EDF) pourrait permettre d'atteindre des niveaux d'incertitudes faibles permettant de les considérer comme des références reconnues, et ainsi se rapprocher de l'étalonnage des $\mathrm{ADCP}$ en rivière sur des gammes de débits et des configurations variées. 


\section{REMERCIEMENTS}

Les auteurs remercient tous les jaugeurs, leurs responsables, les organisateurs, pour leur participation active aux différentes comparaisons interlaboratoires citées dans cette étude. Cette étude est soutenue par le SCHAPI (via la convention Irstea-DGPR) et l'OMM.

\section{RÉFÉRENCES}

AtMANE D. (2012) - Les essais interlaboratoires en hydrométrie : analyse des campagnes de mesures réalisées et amélioration du protocole. Mémoire de fin d'études. Université de Nancy

Chaumont C., Guerin A. (2011) - Comparaison des courantomètres FLO-MATE 2000 de l'unité de recherche HBAN. Rapport technique

Dramais G., Blanquart B., Le Coz J. (2011) - Rapport d'essai : Comparaison de méthode de mesure du débit des petits cours d'eau. Journée d'intercomparaison des 17 et 18 mai 2011. Cemagref

Everard N. (2009) - AdcP RegatTA, River Severn at Bewdley 4th June 2009, [Rapport]. - [s.l.] : UK Environment Agency

Gum 1995 (2008) - Évaluation des données de mesure - Guide pour l'expression de l'incertitude de mesure. Comité commun pour les guides en métrologie JCGM 100. $132 \mathrm{p}$

Hauet A., Le Coz J., Sevrez D., Dramais G., Henault F., Perret C., Pierrefeu G., Pobanz K., Thollet F. (2012) - inter comparaison ADCP sur le canal de la Gentille (12-16/09:2011) rapport technique

Iso 3455. (2007) — Hydrométrie : Étalonnage des moulinets en bassins découverts rectilignes. Norme internationale. $15 \mathrm{p}$

Iso 5725-2 (1994) — Exactitude (justesse et fidélité) des résultats et méthodes de mesure. Partie 2: Méthode de base pour la détermination de la répétabilité et de la reproductibilité d'une méthode de mesure normalisée
Iso 9555-1 (1994) — Méthodes de dilution en régime permanent utilisant des traceurs. Mesure de débit des liquides dans les canaux découverts. 1

Iso 13528 (2005) - Méthodes statistiques utilisées dans les essais d'aptitude par comparaison interlaboratoire

Iso 21748 (2010) — Lignes directrices relatives à l'utilisation d'estimations de la répétabilité, de la reproductibilité et de la justesse dans l'évaluation de l'incertitude de mesure

Le Coz J., Pierrefeu G., Brochot J.F., Saysset G., Marchand P. (2007) - Jaugeage des rivières par ADcp : pour une culture commune. La Houille Blanche. 4 : 111-118

Le Coz J., Saysset G., Pierrefeu G. (2009) - Régate ADCP 1, 3-4-5/02/2009, Vézère au pont de Garavet (Allassac, Corrèze), rapport technique (Groupe Doppler)

Le Coz J., Camenen B., Peyrard X., Dramais G. (2012) Uncertainty in open-channel discharges measured with the velocity-area method. Flow Measurement and Instrumentation. $26: 18-29$

Le Coz J., Pobanz K., Faure. J.B., Pierrefeu G., Blanquart B. (2012) - Stage-discharge hysteresis evidenced by multi-ADCP measurements. RiverFlow 2012, San José

Metinfo (2012) - METAS étalonne des instruments de mesure hydrométrique. Journal de métrologie

Olivier A., Pierrefeu G., Scotti M., Blanquart B. (2009) Incertitudes des mesures de débit réalisées à 1'ADCP. $L a$ Houille Blanche. 3 : 132-137

Pobanz K., Le Coz J., Pierrefeu G. (2011) — Intercomparaison ADCP sur le Rhône à l'aval du barrage de Génissiat (12-15/10/2010). rapport technique (Groupe Doppler)

Terek B., Bušelić G., Nimac N., Bančić S., ŽAlac R., LukaC I. (2008) - Interkomparacijsko mjerenje protoka akustičkim Doppler (ADCP) protokomjerima, Sisak, Hrvatska Rujan [Rapport]. - [s.l.] : DHMZ Zagreb 